\title{
Array Dataflow Analysis for Explicitly Parallel Programs
}

\author{
Jean-François Collard ${ }^{1}$ and Martin Griebl ${ }^{2}$ \\ 1 Laboratoire PRISM, Université de Versailles St-Quentin, 45 Avenue des \\ Etats-Unis, 78035 Versailles, FRANCE, Jean-Francois.Collard@prism.uvsq.fr \\ 2 FMI, Universität Passau, Innstraße 33, 94032 Passau, GERMANY, \\ Martin.Grieb]@fmi.uni-passau.de
}

\begin{abstract}
This paper describes a dataflow analysis of array data structures for data-parallel and/or control- (or task-) parallel imperative languages. This analysis departs from previous work because it 1) simultaneously handles both parallel programming paradigms, and 2) does not rely on the usual iterative solving process of a set of data flow equations but extends array dataflow analysis based on integer linear programming, thus improving the precision of results.
\end{abstract}

\section{Introduction}

After decades of parallel processing, few programming languages can claim to be used on a wide range of parallel architectures. Probably, one of the reasons lies in the difficulty of efficiently compiling a general language on an ever-widening spectrum of machines. Among useful analyses, dataflow analysis derives information about the definition and the subsequent use(s) of data values in a program. Its applications include dead-code elimination, strength reduction, array expansion [1] or equivalently conversion into single-assignment form.

Unfortunately, very few data-flow analyses have been proposed for parallel languages (but see [7]). This paper presents an analysis for data-parallel languages, e.g. HPF [2], control-parallel (also called task-parallel) languages, such as PCF [3], or a mixture of both $[4,5,6]$.

\section{Motivating Examples}

Several languages $[4,6]$ have indexed parallel constructs whose semantics correspond to what we call doall in this paper: a statement instance is spawned for each possible value of the index variable (e.g., from 0 to $2 n$ by step 1 in Program ExD, hence $2 n+1$ instances or tasks are spawned). Each instance has its own copy of shared data structures, and all reads and writes are applied to this copy. Shared data structures are updated only when the instances of all statements in the loop body have completed. Thus, within one iteration of a doall loop, all statements work on the same copy.

For example in Program ExD, if $n=2$, the read of a(1) in Instance number 1 of Statement $R$ may return the value produced by the instance number 1 of 


\begin{tabular}{|c|c|}
\hline$P_{1}$ & $\begin{array}{l}\text { program ExD } \\
\text { doall }(i=0: 2 * n: 1) \\
\text { where }(\ldots)\end{array}$ \\
\hline$S_{1}:$ & $a(i)=\ldots$ \\
\hline$S_{2}:$ & $\begin{array}{l}\text { else } a(2 * n-i)=\ldots \\
\text { endwhere }\end{array}$ \\
\hline$R:$ & $\ldots=a(i)$ \\
\hline
\end{tabular}

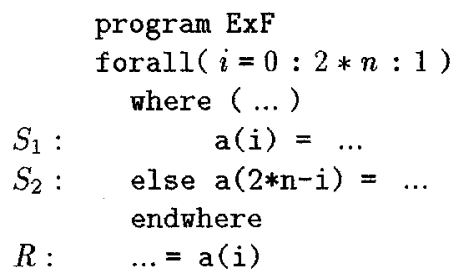

Fig. 1. Two examples

$S_{1}$, but not from Instance 3 of $S_{2}$. Consequently, the information we would like to automatically derive is that the source of a(i) in Instance $i$ of $R$ is either $S_{1}$ in Instance $i$ or undefined (written as $\perp$ ) if $i \neq n$; or: $S_{1}$ or $S_{2}$ in Instance $i$ if $i=n$ (because in this case, either $S_{1}$ or $S_{2}$ writes into a(n)).

Notice that we consider that all instances of both arms of the parallel conditional structure where are executed in parallel.

Similarly, a forall construct spawns as many instances as there are possible values for the index variable (e.g., $2 n+1$ instances are spawned in Program ExF). The semantics of forall we consider is reminiscent from the HPF semantics ${ }^{3}$ [2]: in a multi-statement forall, the array assignment semantics are applied to each statement in turn. Each instance of a statement has its own copy of shared data structures, but these shared data structures are updated before the instances of following statements begin.

Consider the read of a(1) in Program ExF: it may be the case that both $S_{1}$ and $S_{2}$ simultaneously wrote into this cell. Such an over-determined source will be denoted by top (T). To sum up, our analysis derives that the source of a ( $i$ ) in Instance $i$ of $R$ in Program ExF is either $S_{1}$ in Instance $i$ or $S_{2}$ in Instance $2 n-i$ or $\perp$ or $T$, if $i \neq n$, or $S_{1}$ or $S_{2}$ in Instance $i$, if $i=n$ (without $\perp$ nor $\mathrm{T}$ ).

\section{Definitions}

The input language includes the following sequential control structures; do, while, if, the following parallel structures: forall, doall, where, and parallel sections parsection.

The dimension of a vector $\mathbf{x}$ is denoted by $|\mathbf{x}|$. The $k$-th entry of vector $\mathbf{x}$, $k \geq 0$, is denoted by $\mathbf{x}[k]$. The sub-vector built from components $k$ to $l$ is written as: $\mathbf{x}[k . . l]$. If $k>l$, then this vector is by convention the vector of dimension 0 . Furthermore, $\leqslant(\ll)$ denotes the (strict) lexicographical order on such vectors.

The index vector of a statement $S$ is the vector built from the counters of surrounding do, forall, doall and while constructs. An operation is an instance of some Statement $S$, and will be denoted by $\langle S, \mathbf{x}\rangle$, where $\mathbf{x}$ is some value of the index vector of $S$.

\footnotetext{
${ }^{3}$ Note that in HPF the only parallel constructs inside foralls are foralls and wheres (Rules H404 and H406 of [2]).
} 
The depth of a statement or construct is the number of surrounding do, forall, doall or while constructs. So, the depth of $S$ equals to $|\mathbf{x}|$. If $\mathbf{x}[p]$, $p \geq 0$, is a counter of a do, forall or doall construct, then lower and upper affine bounds are known: $l_{p}(\mathbf{x}[0 . . p-1]) \leq \mathbf{x}[p] \leq u_{p}(\mathbf{x}[0 . . p-1])$ where $l_{p}$ and $u_{p}$ are syntactically given by the loop bounds. In the case of while-loops, we have by convention $1 \leq \mathbf{x}[p]$. The index domain of a statement $S$ is denoted by $\mathbf{D}(S)$ and is given by the conjunction of all inequalities on surrounding loop bounds. We define $C_{p}(S)$ as the iterative (do, while, forall or doall) construct surrounding $S$ at depth $p$. (When clear from the context, $S$ will be omitted.) For example, let us consider Statement $S_{1}$ in Program ExD: $C_{0}=P_{1}$ (the doall).

We define $\mathcal{P}(S, R)$ to be the par construct surrounding both $S$ and $R$ such that $S$ and $R$ appear in distinct sections of the par construct. (Notice that there is at most one such construct.) Moreover, let $M_{S R}$ be the depth of $\mathcal{P}(S, R)$. (If $\mathcal{P}(S, R)$ does not exist, then $M_{S R}$ is the number of do, forall, doall or while constructs surrounding both $S$ and $R$.) In Program ExF, $\mathcal{P}\left(S_{1}, R\right)=\emptyset$ and $M_{S_{1} R}=1$. Predicate $\Delta_{S R}$ is true if $S$ and $R$ appear in opposite arms of an if..then. . else or where. .els ewhere construct, or in distinct sections of a par construct. Predicate $T_{S R}$ is true if $S$ textually precedes $R$ and $\Delta_{S R}$ is false. We denote by $\mathcal{W}(u)$ (resp. $\mathcal{R}(u)$ ) the memory cell written into (resp. read) by operation $u$, so for instance $\mathcal{W}\left(\left\langle S_{2}, i\right\rangle\right)=\mathrm{a}(2 n-i)$ and $\mathcal{R}(\langle R, i\rangle)=\mathrm{a}(i)$ in Programs ExD and ExF.

\section{The Semantics: Execution Order}

The purpose of this section is not to give a complete semantical description of a parallel language. As far as dataflows are concerned, we are mainly interested in the order in which computations, and their corresponding writes and reads to memory, occur. The fact that $\langle S, \mathbf{x}\rangle$ is executed before $\langle R, \mathbf{y}\rangle$ in the parallel program will be denoted by $\langle S, \mathbf{x}\rangle \prec\langle R, \mathbf{y}\rangle$. In the case of sequential programs, $\prec$ is a total order which can be expressed as the lexicographical order on index vectors. In turn, the lexicographical order can be expressed as a disjunction of linear inequalities. Expressing the execution order $\prec$ of parallel programs is more intricate. For instance, Section 2 showed that two semantics can be chosen for a data-parallel construct: the "synchronous" semantics of the construct we call forall, where the memory is updated between the execution of two successive statements inside a forall; and the "asynchronous" semantics of the construct we call doall, where none of the spawned tasks sees the effects produced by other tasks. However, thanks to the semantics of forall and doall constructs, $\prec$ can still be expressed in a linear way:

$$
\begin{aligned}
& \langle S, \mathbf{x}\rangle \prec\langle R, \mathbf{y}\rangle \Leftrightarrow\left(\begin{array}{c}
\bigvee_{p=0.1,} \\
C_{p}=\text { do } \vee C_{p}=\text { while }
\end{array}\right. \\
& \vee\left(\left(\bigwedge_{p=0 . . M_{S R^{-1}}} E q u\left(p, C_{p}, \mathbf{x}, \mathbf{y}\right)\right) \wedge T_{S \boldsymbol{R}}\right)
\end{aligned}
$$


where:

$$
\begin{aligned}
& \operatorname{Pred}(p, \mathbf{x}, \mathbf{y}) \equiv\left(\bigwedge_{i=0 \ldots p-1} E q u\left(i, C_{i}, \mathbf{x}, \mathbf{y}\right)\right) \wedge \mathbf{x}[p]<\mathbf{y}[p] \\
& E q u(p, \text { do, } \mathbf{x}, \mathbf{y}) \equiv E q u(p, \text { while }, \mathbf{x}, \mathbf{y}) \equiv \mathbf{x}[p]=\mathbf{y}[p] \\
& E q u(p, \text { forall }, \mathbf{x}, \mathbf{y}) \equiv \operatorname{true} \\
& E q u(p, \operatorname{doall}, \mathbf{x}, \mathbf{y}) \equiv \mathbf{x}[p]=\mathbf{y}[p]
\end{aligned}
$$

Obviously, (1) is a partial order on operations. (In particular, it has no cycle.) Intuitively, Predicate Pred in (1) formalizes the sequential order of a given do or while loop at depth $p$. Such a loop enforces an order up to the first par construct encountered at depth $M_{S R}$ while traversing the nest of control structures, from the outermost level to the innermost. The order of sequential loops is given by the strict inequality in (2), under the condition that the two operations at hand are not ordered up to level $p-1$; hence the conjunction on the $p$ outer predicates $E q u$. Notice that the instances of two successive statements inside a forall at depth $p$ are always ordered at depth $p$ due to (4), but are ordered inside a doall only if they belong to the same task (i.e., the values of the doall index are equal, cf $(5))$.

Note that $\forall P, \bigvee_{i \in \emptyset} P(i)=$ false and $\bigwedge_{i \in \emptyset} P(i)=$ true. Note also that the lexicographical order on nests of do loops [1] and in (sequential) dynamic control programs [8] comes as a special case of (1).

Example As far as programs ExD and ExF are concerned, the order between $S_{1}$ (or $S_{2}$ ) and $R$ is given by:

ExF: $\mathcal{P}\left(S_{1}, R\right)=\emptyset$, so $M_{S R}=1 . C_{0}=$ forall and $T_{S_{1} R}=$ true. Thus:

$$
\begin{aligned}
\left\langle S_{1}, i^{\prime}\right\rangle \prec\langle R, i\rangle & \Leftrightarrow\left(\bigvee_{p \in \emptyset} \operatorname{Pred}\left(p, i^{\prime}, i\right)\right) \vee\left(E q u\left(0, C_{0}, i^{\prime}, i\right) \wedge T_{S_{1} R}\right) \\
& \Leftrightarrow \text { false } \vee(\text { true } \wedge \text { true }) \Leftrightarrow \text { true }
\end{aligned}
$$

This is a formal restatement of a semantical property of foralls, cf Section 2. Notice that $\left\langle S_{2}, i^{\prime}\right\rangle \prec\langle R, i\rangle$ is also always true.

ExD: $\mathcal{P}\left(S_{1}, R\right)=\varnothing$, so $M_{S R}=1$. $C_{0}=$ doall and $T_{S_{1} R}=$ true. Thus:

$$
\begin{aligned}
\left\langle S_{1}, i^{\prime}\right\rangle \prec\langle R, i\rangle & \Leftrightarrow\left(\bigvee_{p \in \emptyset} \operatorname{Pred}\left(p, i^{\prime}, i\right)\right) \vee\left(\operatorname{Equ}\left(0, C_{0}, i^{\prime}, i\right) \wedge T_{S_{1} R}\right) \\
& \Leftrightarrow \text { false } \vee\left(i^{\prime}=i \wedge \text { true }\right) \Leftrightarrow i^{\prime}=i
\end{aligned}
$$

\section{Dataflow Analysis}

Our dataflow analysis first builds the set of possible sources of the flow of a given data, and then selects the latest element, i.e., the maximal element according to the original sequential order. Selecting the maximal element is then done using Integer Linear Programming techniques. 


\subsection{Method Overview}

Let us consider two statements $S$ and $R$. Suppose that $S$ writes into an array a and that $R$ reads that same array:

$$
\begin{array}{ll}
S: & \mathbf{a}(f(\mathbf{x}))=\ldots \\
R: & \ldots=\mathrm{a}(g(\mathbf{y}))
\end{array}
$$

The aim of array dataflow analysis is to find the source of the value $a(g(\mathbf{y}))$ read in $R$ for a given $\mathbf{y}$. This source is denoted by $\sigma(\langle R, \mathbf{y}\rangle)^{4}$. To be a source candidate, an operation $\langle S, \mathbf{x}\rangle$ has to satisfy the following constraints:

Existence predicate: $\langle S, \mathbf{x}\rangle$ is a valid operation: $e(\langle S, \mathbf{x}\rangle)$ evaluates to true. (See Section 5.2.)

Conflicting accesses: $\langle S, \mathbf{x}\rangle$ and $\langle R, \mathbf{y}\rangle$ access the same array cell: $f(\mathbf{x})=$ $g(\mathbf{y}) . f$ and $g$ are possibly multi-dimensional affine functions.

Sequencing condition: $\langle S, \mathbf{x}\rangle$ is executed before $\langle R, \mathbf{y}\rangle$ in the parallel program: $\langle S, \mathbf{x}\rangle \prec\langle R, \mathbf{y}\rangle$. Notice that this leads to a formal definition of $\perp: \perp$ is the name of the operation that is executed once before all other operations of the program, i.e., $\forall S, \mathbf{x}: \perp \prec\langle S, \mathbf{x}\rangle$.

Environment: The set of source candidates is computed under the hypothesis that $\langle R, \mathbf{y}\rangle$ is a valid operation, i.e., $\mathbf{y} \in \mathbf{D}(R)$.

The set of candidate sources is thus:

$$
\begin{array}{r|rr}
\mathbf{Q}_{S R}(\mathbf{y})=\{\langle S, \mathbf{x}\rangle \mid & e(\langle S, \mathbf{x}\rangle), & (\text { Existence }) \\
& f(\mathbf{x})=g(\mathbf{y}), & \text { (Conflicting accesses }) \\
& \langle S, \mathbf{x}\rangle \prec\langle R, \mathbf{y}\rangle\} & (\text { Order })
\end{array}
$$

The direct dependence from $S$ to $R$ is then $K_{S R}(\mathbf{y})=\max _{\prec} \mathbf{Q}_{S R}(\mathbf{y})$. Obviously, $S$ may not be the only statement writing into a. Let $\mathcal{S}(R)$ be the set of statements writing into the array read by $R$. Then, the set of candidate sources is $\mathbf{Q}_{R}(\mathbf{y})=$ $\bigcup_{S \in \mathcal{S}(R)} \mathbf{Q}_{S R}(\mathbf{y})$. The source $K_{R}(\mathbf{y})$ of the flow of datum a $\left.g(\mathbf{y})\right)$ is the maximal element in $\mathbf{Q}_{R}(\mathbf{y})$ according to $\prec$ :

$$
K_{R}(\mathbf{y})=\max _{\prec} \mathbf{Q}_{R}(\mathbf{y}) .
$$

Clearly, three problems occur:

- We have to express Predicate $e$. This issue is addressed in Section 5.2.

- Maxima according to $\prec$ among sets of operations have to be computed. Section 5.3 explains how to compute max $\prec$ using the Omega tool.

- $K_{R}(\mathbf{y})$ may not be uniquely defined, since $\prec$ is not a total order. Intuitively, a non-unique maximum means that two operations wrote in an undefined order into the same memory cell. The over-determined source is denoted by $T$ (cf Section 5.4).

\footnotetext{
${ }^{4}$ For the sake of clarity, we assume that an operation executes at most one read.
} 


\subsection{Existence of Operations}

We say that an operation exists if this operation executes. In the case of staticcontrol sequential programs, the only loops are do loops, and the existence predicate boils down to $e(\langle S, \mathbf{x}\rangle) \Leftrightarrow \mathbf{x} \in \mathbf{D}(S)$.

When arbitrary while, if and where constructs appear, the control flow is dynamic, and existence of operations cannot in general be predicted. The problem then boils down to finding a suitable coding of Existence predicate $e$. The reader is referred to $[8,10]$ for details. In the sequel, we will use the Omega package[9] and phrase this paper in the corresponding framework ${ }^{5}$. For instance, the case of if and where constructs is handled as follows: If $\mathbf{x}$ is the index vector of a conditional statement, the execution of the then branch is coded by postulating that some uninterpreted function $f$ evaluates to, say, a nonnegative value: $f(x) \geq 0$. The execution of the else or elsewhere branch is then denoted by $f\left(x^{\prime}\right)<0$. Since both branches cannot execute in the same instance of the construct, $f(\mathrm{x}) \geq 0 \wedge f\left(\mathrm{x}^{\prime}\right)<0 \Rightarrow \mathrm{x} \neq \mathrm{x}^{\prime}$.

Example Let us find possible bottoms in the source of the read $\langle R, k\rangle$ in Program ExF. ( $k$ is here a parameter.) The set of possible writes from $S_{1}$ is:

\# Omega Calculator [v1.00, Mar 96]:

\# symbolic $n, f(1), k$;

\#W1 :=\{[iw] : $0<=i w<=2 n \& \&($ Set $)>=0 \& \& i w=k\}$;

where $f($ Set $)$ means that $f$ is applied to the bounded variable(s) that define the set (here, iw). Then, for $S_{2}$ :

\#W2 := $\{[i w]: 0<=i w<=2 n \& \& f($ Set $)<0$ \&\& $2 n-i w=k\}$;

We are interested in finding all reads that do not have a corresponding write from either $S_{1}$ or $S_{2}$. We thus take the union of the two sets of writes:

\#W1 union W2;

$\{[i w]: k=i w \& \& 0<=i$ in $<=2 n \& \& 0<=f(i w)\}$ union

$\{[i w]: k+i w=2 n$ \&\& $0<=i w<=2 n \& \& f(i w)<=-1\}$

We then subtract the obtained set to the set $\mathrm{R}$ of all reads:

$\# \mathrm{R}:=\{[\mathrm{ir}]: 0<=$ ir $<=2 \mathrm{n} \& \&$ ir $=\mathrm{k}\}$;

\# Bottoms $:=\mathrm{R}$ intersection complement (W1 union W2);

\# Bottoms ;

$\left\{\left[\operatorname{In}_{-} 1\right]: \mathrm{k}=\operatorname{In}\right.$ 1 \&\& $\left.\mathrm{n}<\operatorname{In}_{-} 1<=2 \mathrm{n} \& \mathrm{f}\left(\operatorname{In}_{-} 1\right)<=-1\right\}$ union

$\left\{\left[I n_{-} 1\right]: k=I n_{-} 1 \& \& 0<=I_{n} 1<n \& \&\left(I_{-} 1\right)<=-1\right\}$

Since nothing is known about $f$, we have to take a conservative approach and assume that any predicate involving $f$ is true. The set of possibly undefined reads (bottoms) is thus given by $\{k: 0 \leq k<n\} \cup\{k: n<k \leq 2 n\}$. Notice that, as expected, the case $k=n$ does not occur, i.e., $\langle S, n\rangle$ is always defined by $S_{1}$ and/or $S_{2}$.

${ }^{5}$ When called with an input file, the Omega calculator (v1.00 dated March 1996) copies the input equations on the standard output, prefixed by the \# sign. 


\subsection{Computing Maxima}

Solving (9), i.e., computing the maximum (maxima), is equivalent to finding the element(s) $K_{S R}(\mathbf{y})$ such that $\neg \exists \mathbf{x} \in \mathbf{Q}_{S R}(\mathbf{y}), \mathbf{x} \neq K_{S R}(\mathbf{y}): K_{S R}(\mathbf{y}) \prec \mathbf{x}$. This expression may have several solutions, since parallel programs have partial execution orders. (See Section 5.4.) Similarly, $K_{R}(\mathbf{y})$ in (9) can be computed by:

$$
\neg \exists\langle S, \mathbf{x}\rangle, e(\langle S, \mathbf{x}\rangle), \mathcal{W}(\langle S, \mathbf{x}\rangle)=\mathcal{R}(\langle R, \mathbf{y}\rangle),\langle S, \mathbf{x}\rangle \neq K_{R}(\mathbf{y}), K_{R}(\mathbf{y}) \prec\langle S, \mathbf{x}\rangle
$$

\subsection{Detecting Over-Determined Sources}

Detection of over-determined sources is done by checking that no two distinct operations satisfy their respective existence predicates, write into the same memory cell, and are not related by $\prec$. Formally: $\operatorname{Error}(u, v) \equiv e(u) \wedge e(v) \wedge u \neq$ $v \wedge \mathcal{W}(u)=\mathcal{W}(v) \wedge \neg(u \prec v) \wedge \neg(v \prec u)$, where $u$ and $v$ are operations. Notice that, in the general case, checking that no dependence is carried by a doall- or a forall-loop is not sufficient.

Example Let us find the over-determined cases in the source of operation $\langle R, k\rangle$ in Program ExF.

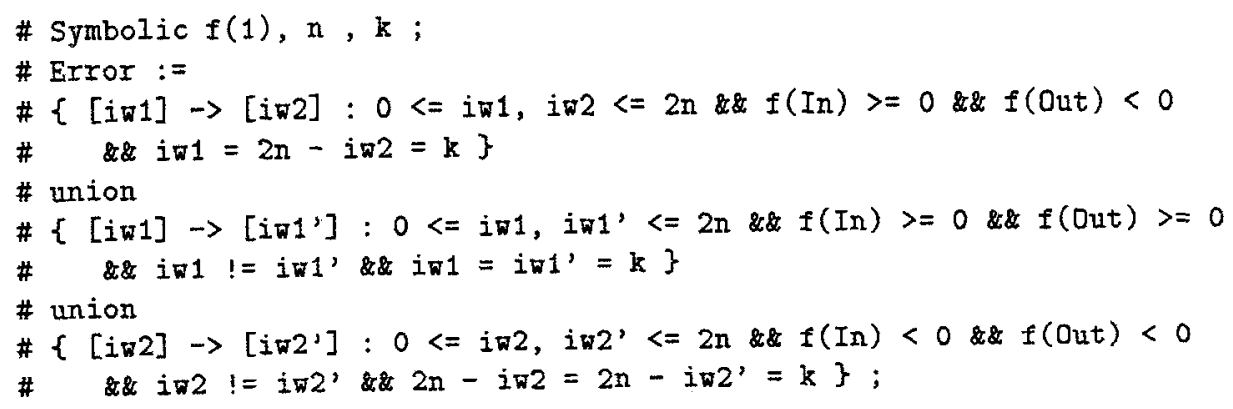

In and Out are collective names of the input (the tuple preceding the $\rightarrow$ and output (the tuple following the $\rightarrow$ ) variables of a relation, respectively.

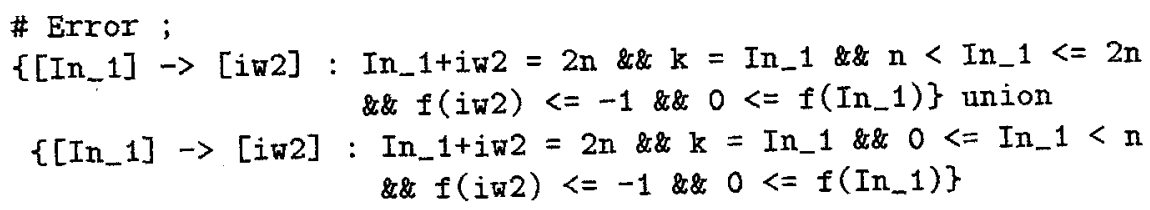

As in the case of bottoms, $f$ is unknown. The set Error of possibly overdetermined reads ( $T$ ) is thus $\{k: 0 \leq k<n\} \cup\{k: n<k \leq 2 n\}$. Notice that this is the result expected from Section 2. 


\section{Conclusion}

This paper presented a dataflow analysis that can be applied to data- and/or task- parallel programs, with dynamic flows of control. Li and Wolfe proposed [11] a simple and precise framework to express the interaction of arbitrarily nested parallel control structures, together with an appropriate data dependence analysis. They did not, however, extended this work to data flows.

The main results of this paper are: a general affine expression for the execution order of programs written in a structured imperative parallel language (this expression subsumes the lexicographical execution order in sequential programs), and a general affine expression for the over-determined cases (Error).

Acknowledgments We would like to thank L. Bougé, T. Brandes, W. Pugh, Y. Robert, G. Utard and M. Wolfe for their helpful comments, and D. Wannacott for his technical help with Omega. Authors are supported by the CNRS and the DFG project RecuR, respectively, and in addition by a German-French programme Procope.

\section{References}

1. P. Feautrier. Dataflow analysis of scalar and array references. Int. Journal of Parallel Programming, 20(1):23-53, February 1991.

2. C.H. Koelbel, D.B. Loveman, R.S. Schreiber, G.I. Stelle Jr, and M.E. Zosel. The High Performance Fortran Handhook. The MIT Press, 1994.

3. Parallel Computing Forum. PCF fortran extensions. Fortran Forum, 10(3), 1991.

4. M. Gerndt and R. Berrendorf. SVM-Fortran, Reference Manual, Version 1.4. KFAZAM-IB-9510, Research Center Jülich May 1995.

5. I. Foster et al. A Compilation System That Integrates High Performance Fortran and Fort ran M. In Proc. of the Scalable High-Performance Computing Conf (SHPCC'9 4). pages 293 300, Knoxville, TN, May 1994.

6. B. Chapman et al. Extending Vienna Fortran with Task Parallelism. In Proc. of the 1994 Intl Conf on Parallel and Distributed System s. pages 258-263, Hsinchu, Taiwan, December 1994.

7. J. Ferrante, D. Grunwald, and H. Srinivasan. Computing communication sets for control parallel programs. In K. Pingali et al., editor, Proc. of $7^{\text {th }}$ Int. W. on Lang. and Compilers for Parallel Comp., volume 892 of LNCS, pages 316-330, Ithaca, NY, August 1994.

8. J.-F. Collard, D. Barthou, and P. Feautrier. Fuzzy array dataflow analysis. In Proc. of 5th ACM SIGPLAN Symp. on Principles and Practice of Parallel Programming, pages 92-102, Santa Barbara, CA, July 1995.

9. W. Pugh. A practical algorithm for exact array dependence analysis. Communications of the ACM, 35(8):27-47, August 1992.

10. W. Pugh and D. Wonnacott. Nonlinear Array Dependence Analysis. In Third Workshop on Languages, Compilers and Run-Time Systems for Sc alable Computers, Troy, NY, May 1992.

11. J. Li and M. Wolfe. Defining, Analyzing, and Transforming Program Constructs. IEEE Parallel and Distributed Technology, 32-39, Spring 1994 